\title{
An approach to improve the performance of the earthquake early warning system for the 2018 Hualien earthquake in Taiwan
}

\author{
Da-Yi Chen ${ }^{1, *}$, Ting-Li Lin ${ }^{2}$, Hsin-Chih Hsu ${ }^{1,3}$, Yi-Chen $\mathrm{Hsu}^{1}$, and Nai-Chi Hsiao ${ }^{1}$ \\ ${ }^{1}$ Central Weather Bureau, Taipei City, Taiwan \\ ${ }^{2}$ Department of Earth Sciences, National Cheng Kung University, Tainan City, Taiwan \\ ${ }^{3}$ Department of Geosciences, National Taiwan University, Taipei City, Taiwan
}

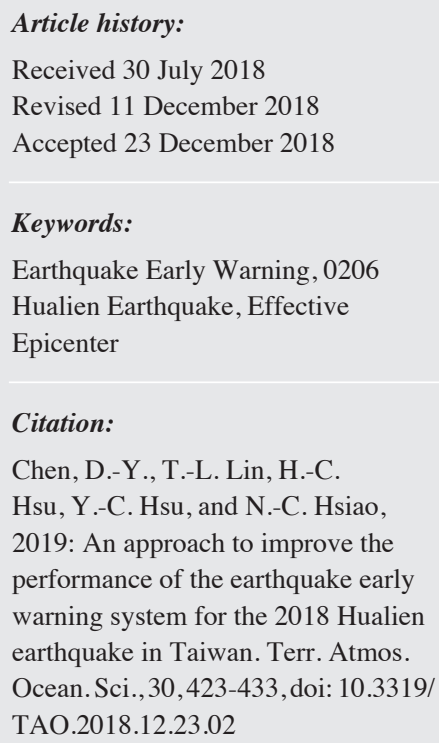

\begin{abstract}
On 6 February 2018, a damaging earthquake with a magnitude of 6.4 on the moment magnitude scale occurred off the eastern coast of Taiwan near Hualien, causing the collapse of buildings and human casualties. About $17 \mathrm{~s}$ after the occurrence of the earthquake, an earthquake alarm was issued by the earthquake early warning (EEW) system operated by the Central Weather Bureau of Taiwan (CWB). During the Hualien earthquake sequence (including foreshocks, mainshock, and aftershocks), the EEW system sent 44 warnings without any false alarms. However, one earthquake with magnitude larger than 5.0 was missed by the EEW system because the multiple earthquakes occurred in a short period causing the EEW system to be unable to correctly distinguish multiple P-wave arrivals. In this study, we proposed an approach that instead uses the centroid of the triggered stations as an effective epicenter and then accordingly calculates magnitude and intensity. The proposed method has been implemented in the new EEW system and was tested during the period of the Hualien earthquake sequence. The results show that the proposed method can shorten the processing time by an average of $4.7 \mathrm{~s}$ as compared to the existing EEW system and can detect all large events without being affected by the multiple concurrent events. In addition, the predicted intensities from the new EEW system were similar to the original intensities.
\end{abstract}

\section{INTRODUCTION}

Taiwan is situated in one segment of the Pacific Rim, which is the most seismically active zone in the world. This seismic activity is dominating by the compression of the Philippine Sea Plate and the Eurasia Plate, two subduction zones located in the eastern and southwestern offshore areas of Taiwan, respectively. Additionally, many active faults are distributed in the inland area of Taiwan. As a result, every year more than 30000 earthquakes occur in Taiwan with local magnitudes larger than 0.5 . Sometimes one of these earthquakes may cause strong shakings and result in property damage and loss of life (Shin and Teng 2001; Rau and Liang 2017). The earthquake early warning (EEW) system, capable of providing warnings before strong ground shaking attacks target areas, is well recognized as a useful tool to mitigate earthquake disasters (Allen et al. 2009).

\footnotetext{
* Corresponding author

E-mail: oceanicdayi@gmail.com
}

Taiwan has developed and tested the EEW system for over two decades. There are four key development stages for the Central Weather Bureau of Taiwan (CWB) operated EEW system. The first stage began with the launch of the system in 1994 in the Hualien area of Taiwan (Wu et al. 1999). In the second stage, the concept of a virtual subnetwork and the $\mathrm{M}_{\mathrm{L} 10}$ method were implemented into an operational system issuing warnings to specific users with a processing time, which is defined as the time between the earthquake origin and the warning issuance, of $22 \mathrm{~s}$ in average (Wu and Teng 2002). In the third stage, the P-wave method was adopted (Wu and Zhao 2006) and the EEW system was developed and tested based on the Earthworm software (Hsiao et al. 2011; Chen et al. 2012), which was used to integrate different kinds of real-time seismic data streams (including broadband, short-period, and strong-motion sensors). The EEW modules based on the Earthworm software were also created and tested. In the fourth stage, a new 
system, named the $e$ BEAR system, has been in operation since 2014 (Chen et al. 2015). In the initial implementation of the $e$ BEAR system the warnings were only sent to schools and specific government agencies. However, since 2016 Taiwan is one of several countries that issue earthquake warnings to the public. The warnings are sent to the public via television broadcasts and cell phone notifications. Figure 1 shows the performance of the $e$ BEAR system from 2014 2017. Due to the geometry of the seismic network, the error of the system in determining the epicenter is approximately 4.1 and $21.0 \mathrm{~km}$ for inland and offshore earthquakes, respectively. The processing time is approximately 17.2 and $26.4 \mathrm{~s}$ for inland and offshore earthquakes, respectively.

The current EEW system has been developed based on the CWB seismic network (CWBSN), shown in Fig. 2. The CWBSN consists of three kinds of seismometers including short-period, broad-band, and strong-motion seismometers. Those instruments are situated either in free-field installations, in boreholes, or on the seabed. Only the amplitudes recorded by the free-field instruments are used for magnitude estimations. However, P-wave arrivals recorded by instruments at all three locations are used to estimate earthquake locations. For the EEW purpose, the length of the data packet should be as short as possible. The CWBSN has upgraded the instruments to make 1-s data packets and to digitize the signal at a 24-bit resolution with the frequency of 100 samples per second.

On 6 February 2018, an $\mathrm{M}_{\mathrm{w}} 6.4$ earthquake violently struck eastern Taiwan and caused large amplitude shaking. The earthquake warning was issued by the CWB EEW system $17 \mathrm{~s}$ after the occurrence of the event. The public received the warning directly from their cell phones or television broadcasts. After 6 minutes, the official earthquake report, which was well checked by the CWB staff, became available. The strong ground-motion caused by the event was recorded by one station with an intensity level of 7 , which indicates a peak ground acceleration (PGA) larger than $400 \mathrm{gal}$. The focal mechanism provided by the CWB indicated that the rupture might have occurred on a steep fault striking either east-southeast, or south-southwest. During the earthquake sequence, the EEW system sent 44 warnings to the public. However, one earthquake with a magnitude larger than 5.0 was missed by the EEW system. The missed event, with a local magnitude of 5.2 occurred about 1 minute after the largest foreshock $\left(\mathrm{M}_{\mathrm{L}} 5.8\right)$ on 4 February 13:57:41. The EEW system failed to distinguish the missed event from the other events because the P-wave arrivals of the multiple events were close together in time.

Two crucial tasks of EEW systems are to predict the seismic intensity level accurately and to reduce the EEW processing time. We propose an approach similar to the concept of the effective epicenter (Teng et al. 1997) to estimate the intensity without the epicenter and earthquake magnitude. This approach does not need to perform any time-con- suming inversion calculation. In the proposed method, the effective epicenter is taken as the average position of the locations of the triggered stations and then used to calculate magnitude and intensity. This proposed effective epicenter method could reduce the processing time. In addition, because of the dense seismic network the epicenter given by the centroid of the triggered stations may not be far away from the true epicenter when the earthquake occurs within the seismic network.

\section{THE CWB EEW SYSTEM}

The EEW system operating in the CWBSN has been developed based on the Earthworm software, which was distributed by the United States Geological Survey in 1994 (Johnson et al. 1995). The Earthworm software is designed to integrate real-time seismic signals coming from different kinds of data formats or data sources. Therefore, the various data sources in the CWBSN, including different kinds of data format, can be integrated by a set of computers running Earthworm in a cluster. Additionally, Earthworm is also suitable for real-time data processing. Either using the official, pre-loaded modules or the modules modified or created by users, Earthworm provides flexible options for system designers. The $e \mathrm{BEAR}$ EEW system, standing for earthwormbased earthquake alarm reporting system, has been created and operated in Taiwan since 2014 (Chen et al. 2015).

\subsection{System Configuration}

Earthworm is flexible for users to design different system configurations for different purposes. Modules and shared memory are the two main components of the Earthworm software program. A module is a program to access and process data. Shared memory is a physical computer memory space where modules can extract and save information. Based on the characteristics of the Earthworm software program, Chen et al. (2015) created a new EEW system named $e \mathrm{BEAR}$. Figure 3 shows the system configuration of the system in which only the vertical component of the seismic waveforms are used. The system can be divided into three major parts including data feeding, data processing, and EEW report generating. For data input, seismic data streams from different types of sources are transferred to the shared memory to provide the waveform data. The phase picking and source parameter estimating are in the data processing part. The phase picking procedure not only picks the P-wave arrivals but also obtains the peak amplitude in displacement $\mathrm{P}_{\mathrm{d}}$ of the P-waves. Subsequently, the source parameter estimating procedure uses the parameters from the phase picking procedure to produce earthquake messages detailing the earthquake origin time, location and magnitude. Finally, the decision making procedure filters the earthquake messages according to certain predefined 

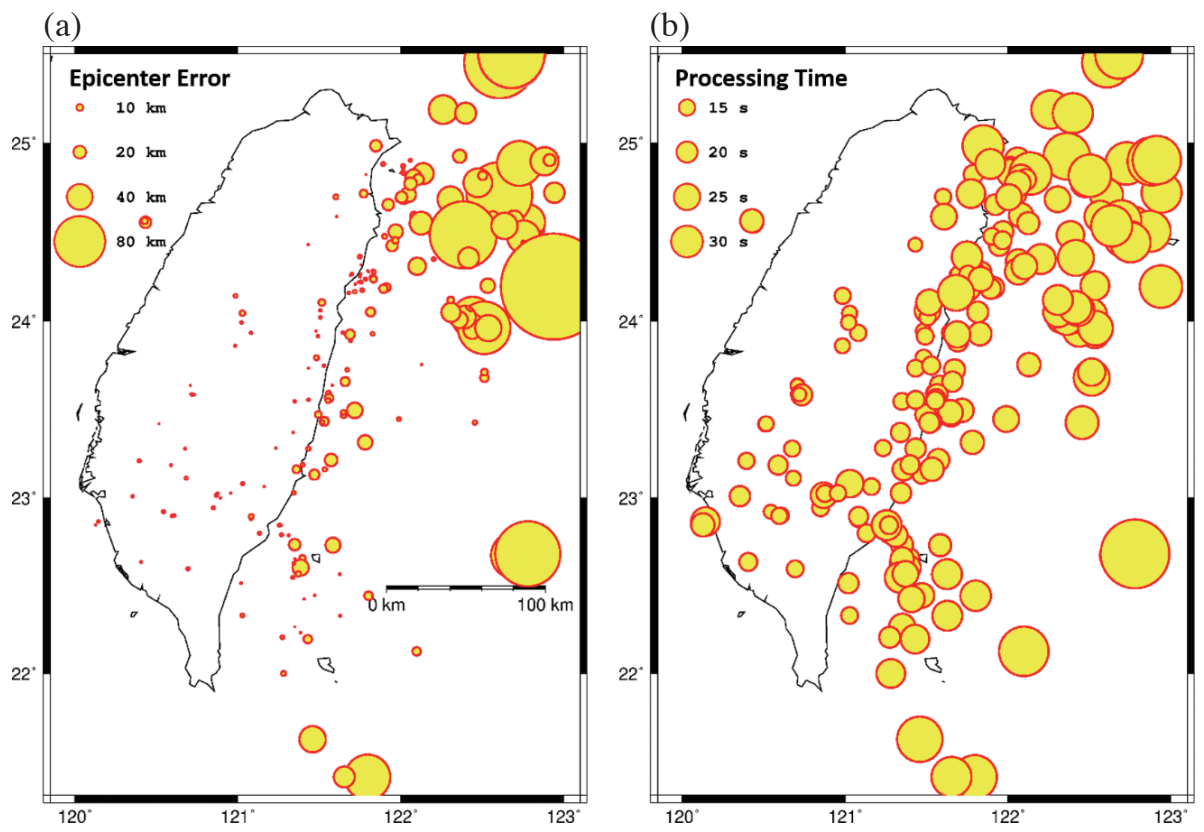

Fig. 1. The performance of the EEW system from 2014 - 2017 compared to the earthquake catalog made by the CWB. (a) The epicenter error, (b) the processing time.

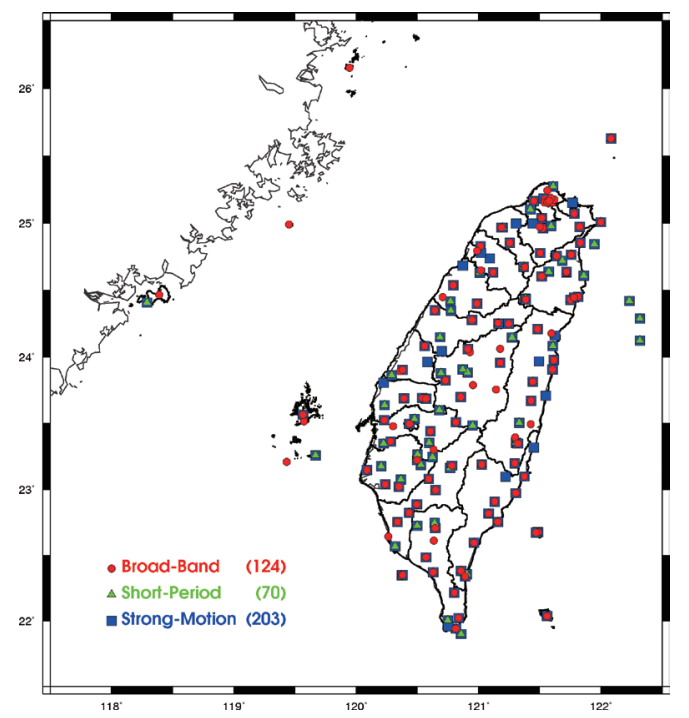

Fig. 2. The station distribution of the CWB seismic network. Red circles represent broad-band sensors; green triangles represent short-period sensors, and blue squares represent strong-motion sensors. The number inside the parentheses is the number of seismic stations.

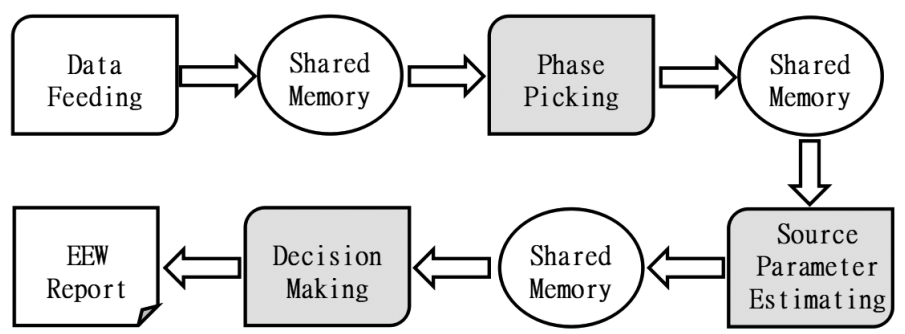

Fig. 3. The configuration of the EEW system. The three shaded gray squares represent Earthworm modules created by the CWB staff for the use in the EEW system. 
thresholds, such as minimum estimated magnitude, intensity and so on, and then the selected EEW reports are available for issuance to the users.

\subsection{Earthquake Location}

In order to estimate earthquake locations rapidly and prevent wrong $\mathrm{S}$-wave arrivals from affecting the location procedure, only $\mathrm{P}$-wave arrivals are used in the EEW system. When detecting P-wave arrivals of an event, the short-term average (STA) over long-term average (LTA) approach is adopted (Allen 1978) on the vertical components of seismic instruments. In order to verify the quality of each pick, when the system detects a P-wave arrival, the first onesecond window after the arrival is used for estimating the signal-to-noise ratio, and the number of zero crossings in the waveform. Both of them are used to evaluate the quality of the arrival. Additionally, in order to avoid having the background noise affect the detection, the system checks the maximum amplitudes of velocity and acceleration within the first two-seconds of the P-wave arrival. If the noise level of the waveform recorded in the station was identified, this procedure can be useful to eliminate false picks from background noise. In order to avoid picking too many picks in the same channel, the picking procedure is stopped for $20 \mathrm{~s}$ after the pick has been declared.

P-wave arrivals generated by triggered stations can be grouped by time and distance. If the number of triggered arrivals is larger than 5 , then the system will start to locate the event using Geiger's method (Geiger 1912). The earthquake location is estimated by using Geiger's method to determine the epicenter and using a grid search method with $10 \mathrm{~km}$ intervals to determine the focal depth. For the determination of the earthquake location, the system uses a half space velocity model to calculate travel times. The onedimensional velocity model was obtained from averaging the three-dimensional model (Wu et al. 2009). For depths shallower than $40 \mathrm{~km}$, the velocity can be found via

$V(D)=5.103+0.067 \times D$

while for depths deeper than $40 \mathrm{~km}$, the velocity can be found via

$V(D)=7.805+0.005 \times D$

where $V(D)$ is velocity in $\mathrm{km} \mathrm{s}^{-1}, D$ is depth in $\mathrm{km}$.

\subsection{Magnitude Estimation}

After the first pick has been declared, the amplitude of each pick is used for magnitude estimation. The amplitude updates by expanding the P-wave time window. To keep the system efficient and to prevent magnitude underestimations, the amplitude is extracted from an expanded P-wave time window of $2-9 \mathrm{~s}$ (Chen et al. 2017). However, in the magnitude estimations the system uses the same equations for the different P-wave time windows. The regression equations are as follows (Chen 2015).

For the broadBand sensor,

$M_{p d}=5.000+1.102 \times \log 10\left(P_{d}\right)+1.737 \times \log 10(R)$

For the acceleration sensor,

$M_{p d}=5.067+1.281 \times \log 10\left(P_{d}\right)+1.760 \times \log 10(R)$

For the short-period sensor,

$M_{p d}=4.811+1.089 \times \log 10\left(P_{d}\right)+1.738 \times \log 10(R)$

In Eqs. (3) - (5), $M_{p d}$ is the magnitude, $R$ is the hypocentral distance in $\mathrm{km}$, and $P_{d}$ is the peak displacement in $\mathrm{cm}$.

To avoid the magnitude estimation being affected by extremely large or small amplitudes, the system will eliminate the extremes and then take the average of all remaining values.

\subsection{Intensity Estimation}

The CWB intensity scale has 8 degrees $(0-7)$, each representing different ground shaking levels. For example, intensity 0 , which relates to a PGA value less than $0.8 \mathrm{~cm}$ $\mathrm{s}^{-2}$, indicates that people cannot feel the shaking. However, intensity 7 may indicate shaking strong enough to cause buildings to collapse. Based on the Weber-Fechner law which relates physical changes and human responses, the calculation of the intensity scale in Taiwan is entirely based on PGA. The intensity can be estimated as

$\log _{10}(\alpha)=(I / 2)-0.6$

where $\alpha$ is the PGA in $\mathrm{cm} \mathrm{s}^{-2}$, and $I$ is the intensity scale.

According to the Eq. (6), an intensity of 6 has a wide range of PGA values, from $250-800 \mathrm{~cm} \mathrm{~s}^{-2}$. Learning from the $1999 \mathrm{M}_{\mathrm{w}}$ 7.6 Chi-Chi earthquake (Shin and Teng 2001), the CWB found that this wide range may cause emergency operation centers to have difficulties in distinguishing the locations of the most damaged areas. Based on the dense strong-motion free-field records of the Chi-Chi earthquake, the CWB found $400 \mathrm{~cm} \mathrm{~s}^{-2}$ is a good indicator for serious damage such as collapsed buildings. Therefore, $400 \mathrm{~cm} \mathrm{~s}^{-2}$ was set as a threshold level. If the measured PGA value is 
larger than $400 \mathrm{~cm} \mathrm{~s}^{-2}$, the shaking will be recognized as at an intensity of 7 .

In the EEW system, after the earthquake parameters are available (including epicenter, depth, and magnitude), it is important to reasonably predict the intensity for areas far away from the epicenter. The CWB EEW system uses the following equation to predict intensity (Hsiao 2006).

$\mathrm{PGA}=1.657 \times e^{1.533 \times M} \times r^{-1.607} \times S_{i}$

where $M$ is the magnitude estimated from the EEW system, $r$ is the hypocentral distance express in $\mathrm{km}, S_{i}$ is the site effect factor, and $i$ indicates different sites.

\section{DECISION MAKING AND WARNING DISSEMINATION}

For the EEW system to begin dissemination of the warning message, a criterion of at least $6 \mathrm{P}$-wave arrivals must be met for both inland and offshore earthquakes. Additionally, when the stations coverage gap is larger than 180 degrees, the minimum required $\mathrm{P}$-wave arrivals increases to 11 . To increase the accuracy of the information in the EEW alarm, the first two warning messages are not considered so as to allow sufficient evaluation of the event details and to reduce the frequency of false or incorrect alarms. The third earthquake message is thus considered as the first EEW alarm.

There are three approaches to rapidly transmit alarm messages to the end users, each with different criteria. The first approach is that EEW alarms will be sent directly from the CWB to the schools, government agencies, and private companies when at least one city with the predicted intensity larger than 2 and when the magnitude is larger than or equal to 4.5. The school and government agencies will then use their public broadcasting systems to warn people inside the buildings. Private companies will have created some device or program for their users to receive warnings. The second approach is that EEW alarms will be sent to television companies for display on their news program when the event has a magnitude larger than or equal to 5.0 and at least one city with the predicted intensity larger than 2 . The third approach is that EEW alarms will be sent out to the public by cell phone in cities where the predicted intensity is larger than 3 (for Taipei city is larger than 2) and when the magnitude is larger than or equal to 5.0.

\section{PERFORMANCE OF THE EEW SYSTEM DURING THE HUALIEN EARTHQUAKE SEQUENCE}

Figure 4a shows the time history of the warning issuances of the mainshock. The CWB EEW system was triggered by the $\mathrm{M}_{\mathrm{w}} 6.4$ earthquake when station TWD detected the initial P-wave at 15:50:44.74. The origin time of the event is at 15:50:42.60. The first earthquake message was issued at 15:50:56.29 using 7 stations with a station coverage gap of 219 degrees. As the seismic waves of the event propagated away from the epicenter, more P-waves became available. The EEW system kept updating the messages. Because the event was located in a near offshore area, the station coverage gap was larger than 180 degrees. The EEW system needed to wait until $11 \mathrm{P}$-waves arrived. Finally, the decision-making module chose the fifth earthquake message as the warning report. The warning was issued $17 \mathrm{~s}$ after the earthquake occurred. The blind zone of this event was about $55 \mathrm{~km}$. Comparing the observed and predicted intensities, shown in Figs. $4 \mathrm{~b}$ and c, the EEW system had underestimated the predicted intensities in the areas near the epicenter. However, the EEW system had issued the warnings in time and provided 5 - $10 \mathrm{~s}$ of lead time for the areas with high intensities. The limitation of an EEW system is that it is impossible for warnings to be issued quickly and also accurately. For emergency response, the accurate earthquake information can be provide by the earthquake rapid reporting system which is more correct but time consuming. For the EEW system, it is necessary to issue alarms as soon as possible. Although the accuracy may be poor, the basic requirements are that the EEW system cannot overestimate intensities.

Observed ground shaking from stations during an earthquake can be used to distinguish between true and false alarms (Kuyuk et al. 2015). The CWB EEW system calculates peak ground acceleration values, defined as $\mathrm{P}_{a}$, within the early portion of the $\mathrm{P}$-wave. The time window for calculating $\mathrm{P}_{\mathrm{a}}$ moves sequentially by wave. The distribution of $\mathrm{P}_{\mathrm{a}}$ given by the EEW system allows the system to understand the shaking distribution and the spatial pattern of the distribution which may indicate rupture direction. Figure 5 shows the $\mathrm{P}_{\mathrm{a}}$ distribution of the Hualien main shock. The first earthquake message was issued $13 \mathrm{~s}$ after the occurrence, and the maximum $\mathrm{P}_{\mathrm{a}}$ value was larger than 70 gal, which means large ground shaking has struck the epicenter area. The second earthquake message, given $14 \mathrm{~s}$ after the occurrence of the event, indicated that the ground shaking was concentrated toward the southern direction. It implied the rupture direction and the potential area for the occurrence of aftershocks. Figures $5 \mathrm{e}$ and $\mathrm{f}$ indicate that the strong shaking was concentrated on the southern part first, and then moved to the northern part. The real-time evolution of the shaking distribution revealed by a high density seismic network may provide information about the rupture process of the mainshock.

Figure 6 presents the performance of the EEW system during the Hualien earthquake sequence. When compared to the CWB earthquake catalog, the location discrepancy of epicenters is about $3.9 \pm 2.7 \mathrm{~km}$, and the magnitude discrepancy is about 0.2 . Although the EEW system now can provide accurate estimations for the Hualien earthquake sequence, the processing time, which was about $20.1 \pm 3.1 \mathrm{~s}$ 
on average, was too long for the EEW purpose. We need provide some solutions to this problem.

\section{EFFECTIVE EPICENTER}

In order to better shorten the processing time in the EEW system, we proposed an effective epicenter method that uses the centroid of the triggered stations as the epicenter and assumes a focal depth of $10 \mathrm{~km}$. This is not the initial rupture point but it can represent the most damaged location. Based on the size of the area with the highest shaking, the effective magnitude can be estimated (Teng et al. 1997). This concept was further implemented (Lin and Wu 2010; Lin et al. 2011) using the area of PGA and $P_{d}$. In order to incorporate this concept into the current EEW system (Chen et al. 2015), the picking information of the EEW system is used and the epicenter is given by the centroid of triggered stations; the magnitude is estimated using the effective epicenter and the $P_{d}$ values. To keep the effective epicenter and magnitude related to the highest shaking, at least 6 triggered stations are needed. For estimating earthquake magnitude from the effective epicenter, the empirical formula, Eqs. (3) - (5), were used. For the EEW system, the key information includes area and intensity. The system should issue EEW messages in time and before the shaking arrive. According to the Eqs. (6) and (7), the proposed method is to provide areas with high intensities, as estimated by the effective epicenter and effective magnitude.

Figure 7 shows the operation procedure of the effective epicenter method. When an earthquake occurs, seismic

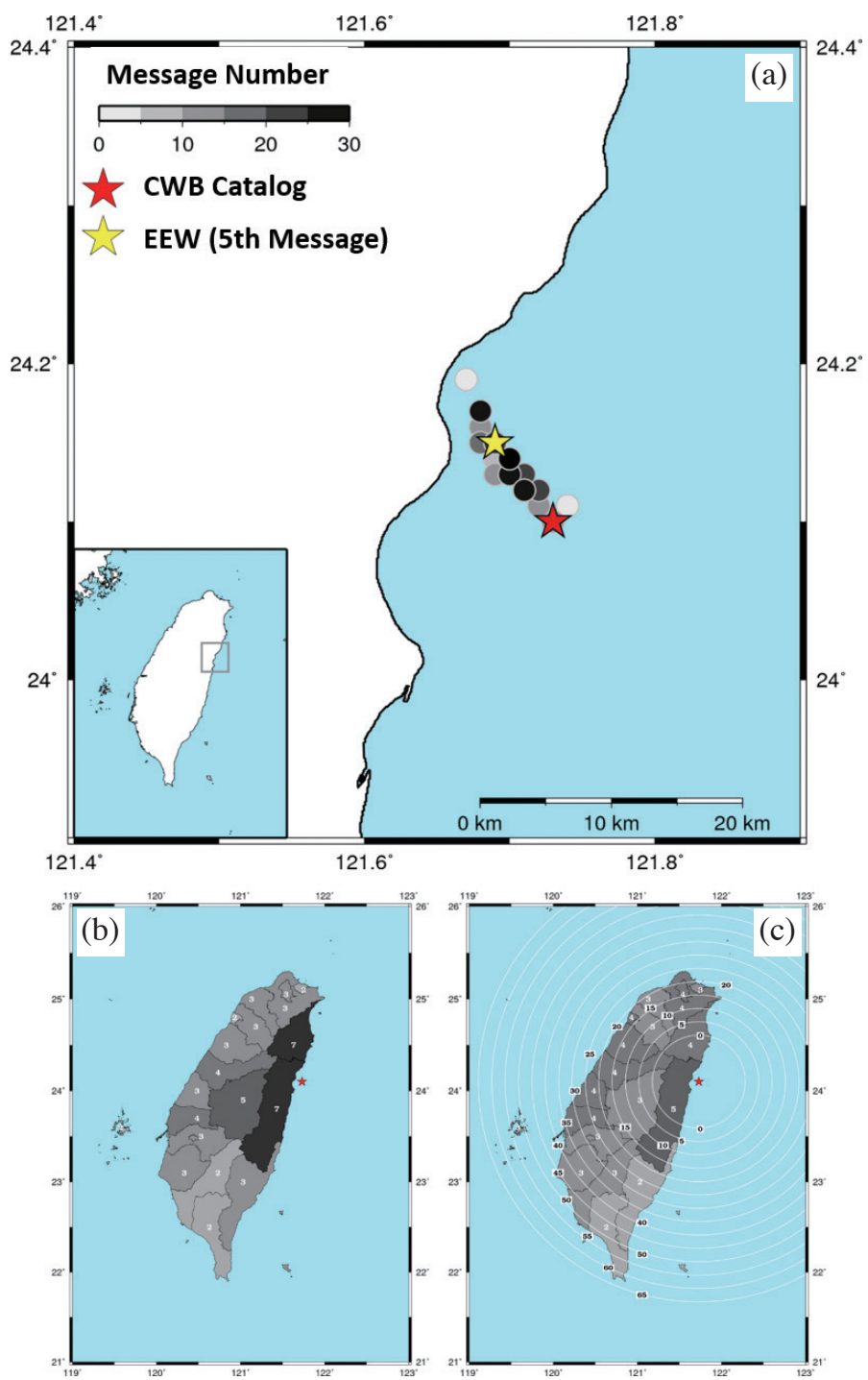

Fig. 4. (a) The location evolution of the earthquake message from the EEW system, with the yellow star representing the fifth message generated by the EEW system and also considered as the first EEW report issued to the public, and the red star representing the epicenter as determined by the CWB staff, (b) the observed intensity of each city or county, (c) the predicted intensity of each city or county. The circle shows the warning time for different areas. The red star is the epicenter of the Hualien main shock with local magnitude 6.2. 

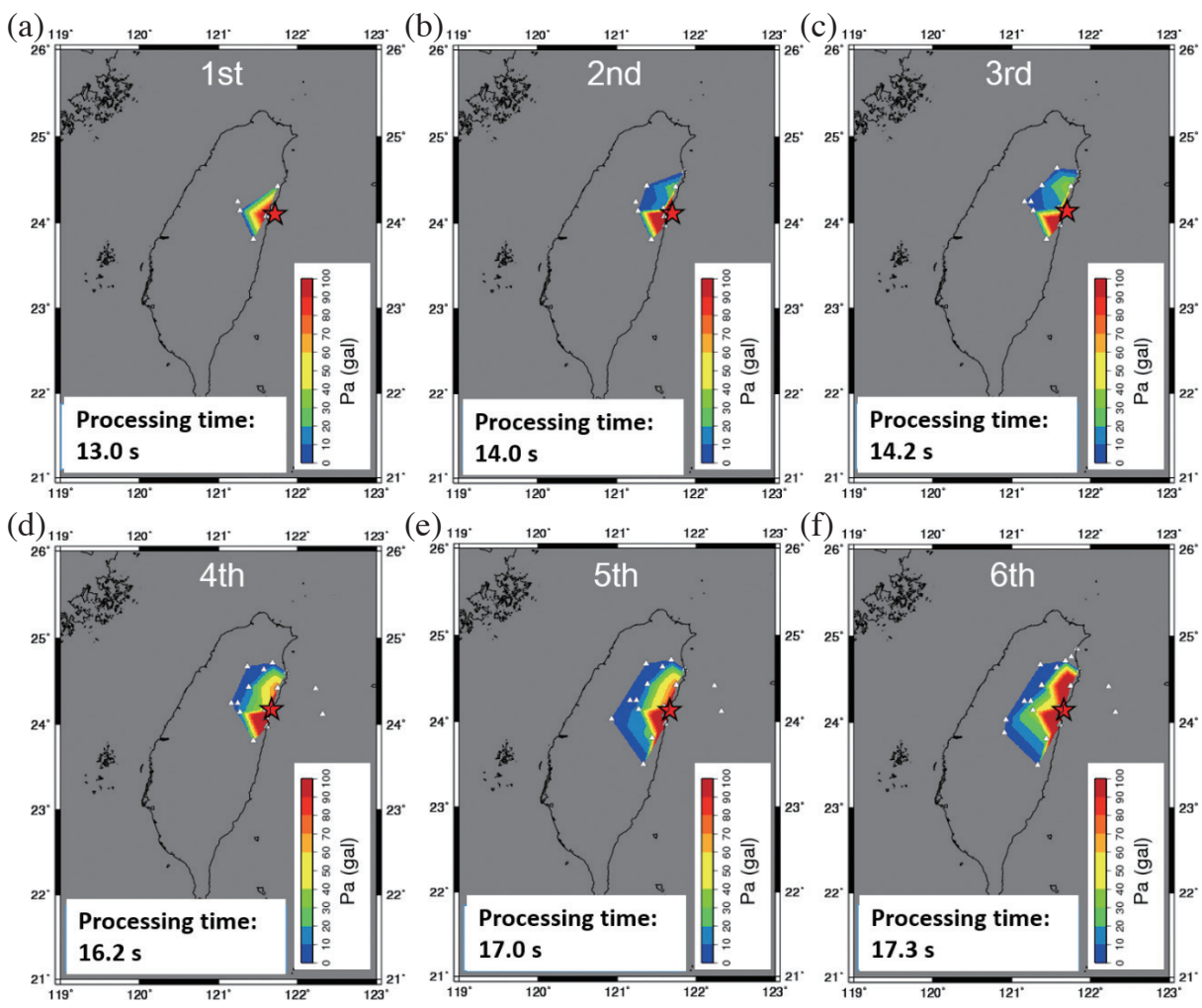

Fig. 5. The distribution of peak acceleration $\mathrm{P}_{\mathrm{a}}$ generated from the original EEW system.
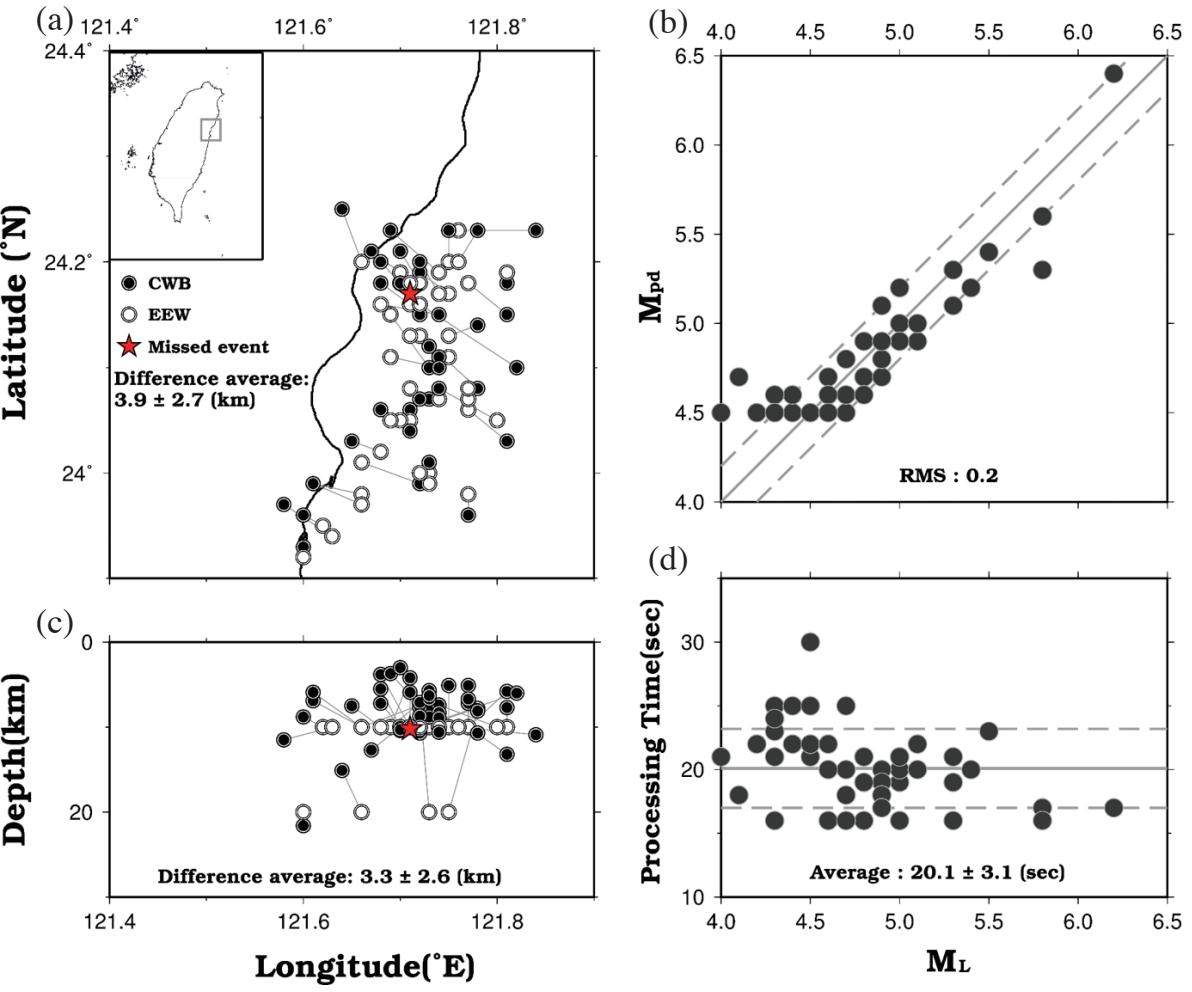

Fig. 6. The performance of the original EEW system, which issued 44 warnings, during the Hualien earthquake sequences (including the foreshocks, main shock, and aftershocks). For the earthquakes with a magnitude larger than 5.0, the EEW system missed one event which is represented by red star. The results from the EEW system were compared to the earthquake catalog made by the CWB staff. The solid circle represent the locations from the earthquake catalog. The open circle represents the locations from the original EEW system. (a) Epicenter error, (b) Depth error, (c) Magnitude error, (d) Processing time. 

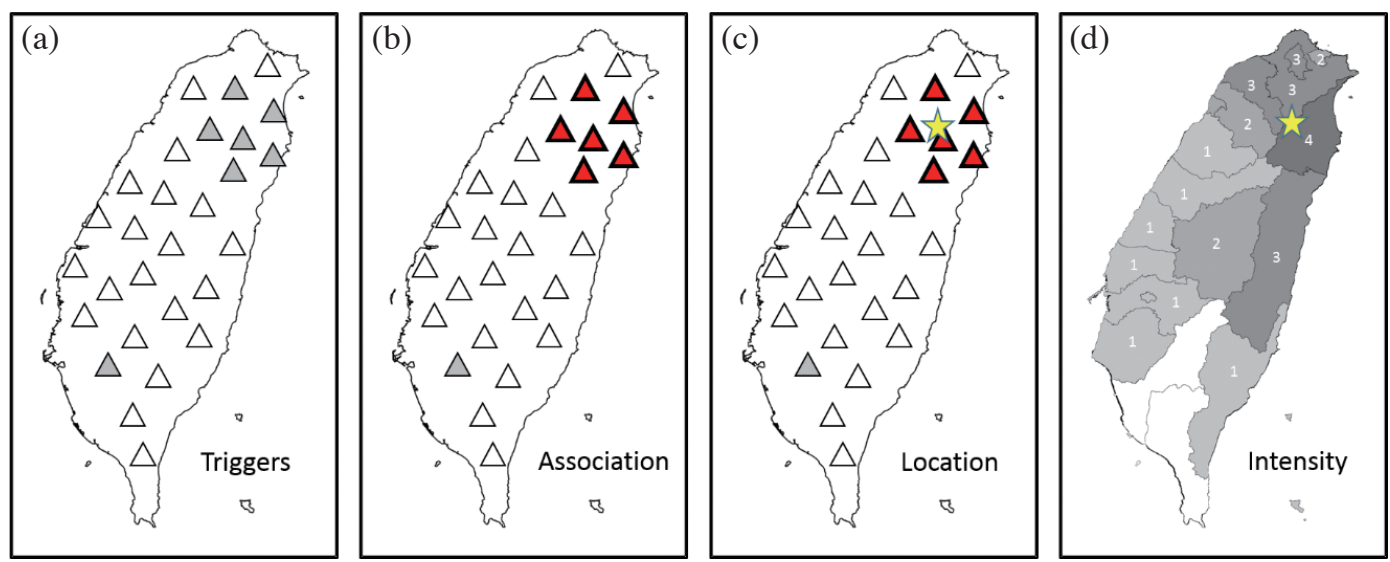

Fig. 7. The operation procedure of the effective epicenter method. (a) The gray solid triangles show the triggered stations, (b) the red solid triangles shows the stations associated by the system, (c) the effective epicenter, determined as the centroid of the associated stations, (d) the estimated magnitude and intensities, found after the effective epicenter is determined.

waves propagate away from the epicenter. The EEW system detects the $\mathrm{P}$ arrivals. A station receives this arrival is considered as a triggered station. Although some stations are triggered by noise, the false triggered arrivals are removed by an associated procedure. The associated procedure is to group associated $\mathrm{P}$ arrivals by considering certain space and time range. In the CWB EEW system, the associated time window is $40 \mathrm{~s}$ for each P-wave arrival and the associated distance is $180 \mathrm{~km}$ for each triggered station. Therefore, in Figs. 7a and b, one triggered station located on south-western Taiwan was eliminated.

The largest difference between the traditional method and the new proposed method is the location procedure. The traditional method uses Geiger's method to locate events which may take more time to obtain results because the travel-time residuals from the method must be less than 0.8 during the calculation. However, the effective epicenter method is more efficient in that it simply calculates the average positions from the triggered stations. When the location is determined, the estimates of magnitude and intensities follow subsequently.

Table 1 shows the comparison of the performance from the effective epicenter method and the traditional method (Geiger's method). To compare against the manually processed earthquake parameters from the CWB, 15 events with magnitudes larger than 5.0 in the Hualien earthquake sequence were selected. The intensity score, which represents the proportion of the correct predictions among all predictions (Kodera et al.2016), is defined as

Intensity Score $=\frac{\text { Number of Correct Prediction }}{\text { Number of Prediction }}$

where the correct prediction means that seismic intensity predicted by the EEW system was within one intensity unit of the CWB observed intensity. In consideration of earthquake hazards, only observed or predicted intensities larger than intensity 2 were considered.

Using the effective epicenter method, the processing time is on average about four seconds faster than when using the traditional method. Although the error in the epicenter from the effective method is larger than that from the traditional method, the intensity score from the new and old method were similar. For earthquakes occur outside the seismic network, the new method usually provides epicenters close to the triggered stations, which means the hypocentral distance is small and eventually the estimated magnitude is small too. On the other hand, comparing to the new method, the old method provides epicenter more close to the true location, which means the hypocentral distance and estimated magnitudes are larger than those given by the new method. However, based on the Eq. (7), smaller hypocentral distance and smaller magnitude may result in similar estimated PGA as to larger hypocentral distance and larger magnitude. Therefore, in Eq. (6), the estimated intensities from provided by the new method were similar to those by the old method.

The effective method can also detect individual events without being affected by the occurrence of multiple concurrent events. This is because the effective method provides the effective epicenter directly by using the triggered stations, whereas the traditional method implements an inversion procedure in which the P-wave arrivals coming from multi-events may enlarge the travel time residuals. In the current EEW system, earthquakes with a high travel time residual might be removed and hence become a missed event.

When two earthquakes occur within one minute of each other, an EEW system that is based on the traditional method may not detect the second earthquake. The $\mathrm{P}$ arrivals coming 
Table 1. The comparisons between the old EEW system (Geiger's method) and new EEW system (effective epicenter method). During the Hualien earthquake sequence, there were 15 earthquakes with local magnitude larger than 5.0. The old EEW system missed one earthquake because multievents occurred in a short time. The new EEW system shows fast processing time, accurate intensity scores, and no missed detections.

\begin{tabular}{|c|c|c|c|c|c|c|c|c|c|c|c|c|c|}
\hline \multirow{2}{*}{ No. } & \multirow{2}{*}{$\begin{array}{c}\text { Date } \\
(\mathbf{m m} / \mathbf{d d})\end{array}$} & \multirow{2}{*}{$\begin{array}{c}\text { Time } \\
\text { (hh:mm:ss) }\end{array}$} & \multirow{2}{*}{$\begin{array}{l}\text { Depth } \\
(\mathbf{k m})\end{array}$} & \multirow{2}{*}{$\mathbf{M}_{\mathbf{L}}$} & \multirow{2}{*}{$\begin{array}{c}\text { Max. } \\
\text { Intensity }\end{array}$} & \multicolumn{2}{|c|}{ Processing Time } & \multicolumn{2}{|c|}{ Intensity Score } & \multicolumn{2}{|c|}{ Epicenter Error } & \multicolumn{2}{|c|}{ Max. Intensity } \\
\hline & & & & & & OLD (sec) & NEW (sec) & OLD (\%) & NEW (\%) & OLD (km) & NEW (km) & OLD & NEW \\
\hline 1 & $02 / 04$ & $21: 12: 52$ & 3.0 & 5.3 & 5 & 16 & 14 & 84 & 80 & 4.0 & 18.1 & 5 & 4 \\
\hline 2 & $02 / 04$ & $21: 56: 41$ & 10.6 & 5.8 & 5 & 16 & 13 & 96 & 92 & 6.8 & 28.9 & 5 & 5 \\
\hline 3 & $02 / 04$ & $21: 57: 42$ & 10.2 & 5.2 & 5 & & 14 & & 87 & & 20.4 & & 5 \\
\hline 4 & $02 / 04$ & $22: 13: 12$ & 10.3 & 5.5 & 5 & 23 & 14 & 96 & 88 & 1.1 & 36.4 & 5 & 4 \\
\hline 5 & $02 / 05$ & $23: 58: 41$ & 8.3 & 5.0 & 5 & 21 & 14 & 100 & 91 & 4.0 & 17.8 & 4 & 4 \\
\hline 6 & $02 / 06$ & 13:09:14 & 10.7 & 5.0 & 5 & 16 & 14 & 82 & 93 & 4.0 & 17.6 & 4 & 4 \\
\hline 7 & $02 / 06$ & $23: 50: 42$ & 6.3 & 6.2 & 7 & 17 & 13 & 82 & 87 & 7.1 & 21.1 & 7 & 5 \\
\hline 8 & $02 / 07$ & 02:00:13 & 6.7 & 5.3 & 4 & 19 & 15 & 88 & 93 & 2.5 & 25.8 & 4 & 4 \\
\hline 9 & $02 / 07$ & 02:07:38 & 4.2 & 5.3 & 4 & 21 & 16 & 100 & 93 & 2.5 & 34.5 & 4 & 3 \\
\hline 10 & $02 / 07$ & $03: 15: 29$ & 5.7 & 5.4 & 4 & 20 & 16 & 92 & 100 & 1.6 & 35.7 & 5 & 4 \\
\hline 11 & $02 / 07$ & 11:36:19 & 7.4 & 5.1 & 3 & 20 & 14 & 100 & 100 & 7.4 & 32.9 & 4 & 3 \\
\hline 12 & $02 / 07$ & 19:13:06 & 8.2 & 5.0 & 4 & 20 & 16 & 91 & 90 & 0.1 & 27.8 & 4 & 4 \\
\hline 13 & $02 / 07$ & 21:06:41 & 7.2 & 5.1 & 4 & 22 & 13 & 100 & 100 & 1.1 & 22.2 & 4 & 4 \\
\hline 14 & $02 / 07$ & $23: 21: 31$ & 7.8 & 5.8 & 4 & 17 & 14 & 86 & 75 & 8.7 & 23.3 & 5 & 5 \\
\hline 15 & $02 / 08$ & 08:54:16 & 11.5 & 5.0 & 4 & 19 & 16 & 90 & 83 & 6.5 & 10.5 & 5 & 5 \\
\hline \multicolumn{6}{|c|}{ Average } & 19.1 & 14.4 & 91.9 & 90.1 & 4.1 & 24.9 & & \\
\hline
\end{tabular}

from the first event can be processed correctly. However, the $\mathrm{P}$ arrivals from the subsequent event could mix with the ones from the prior event. Consequently, the EEW system fails to issue alarms for the second event. Figure 8 shows the waveforms of the event missed event by the use of the traditional method during the Hualien earthquake sequence. The two events occurred within one minute of each other at almost the same location.

\section{DISCUSSION AND CONCLUSIONS}

The 2018 Hualien earthquake caused a series of earthquakes, thus providing a good opportunity to check the current EEW system and test the effective epicenter method. The effective epicenter method can provide predicted intensities with reasonable accuracy and with shorter processing time. In practice, the EEW system can perform the effective method and the traditional method simultaneously. When an earthquake occurs, the effective method is able to provide earlier warning and the traditional method can subsequently update the warning if necessary.

The effective epicenter method may not be seen as an improvement over the traditional method but rather a method that shortens the warning time at the expense of accurately determining the earthquake location and intensity distributions. However, it improved the performance of the previous EEW system. The predicted intensity according to this epicenter is still valuable information for the EEW system. In terms of practical earthquake hazard mitigation strategy, the effective epicenter could be recognized as a secondary earthquake source location and the initial stations not only provide the effective epicenter but also the ground shaking levels near the effective epicenter.

When using the effective epicenter method, earthquakes originating far away from the triggered stations may cause a large error in location. For example, in the case of deep earthquakes or offshore earthquakes, the effective epicenter with depth of $10 \mathrm{~km}$ (assuming the depth is $10 \mathrm{~km}$ ) may be close to the triggered stations yet far away from the actual hypocenter. The magnitude estimations from the effective epicenter will usually be either similar to the actual epicenter or underestimated. In this proposed design, if the magnitude is underestimated, the actual will still cause large shaking, thus the EEW system should issue warnings because the real magnitude is larger than the estimated one. For earthquakes that occur inside the seismic network, the effective epicenter should be close to the real epicenter because the seismic network is very dense in Taiwan.

The centroid of the triggered stations represents the most damaged area, instead of the epicenter used in the conventional method, which typically represents the nucleation point of the event. When compared to the research of Teng et al. (1997), the method proposed here is more efficient as a result of the $\mathrm{P}_{\mathrm{d}}$ values being determined from the evolutionary $\mathrm{P}_{-}$ wave time window. The next-generation configuration of the CWB EEW system has been designed and tested recently. 

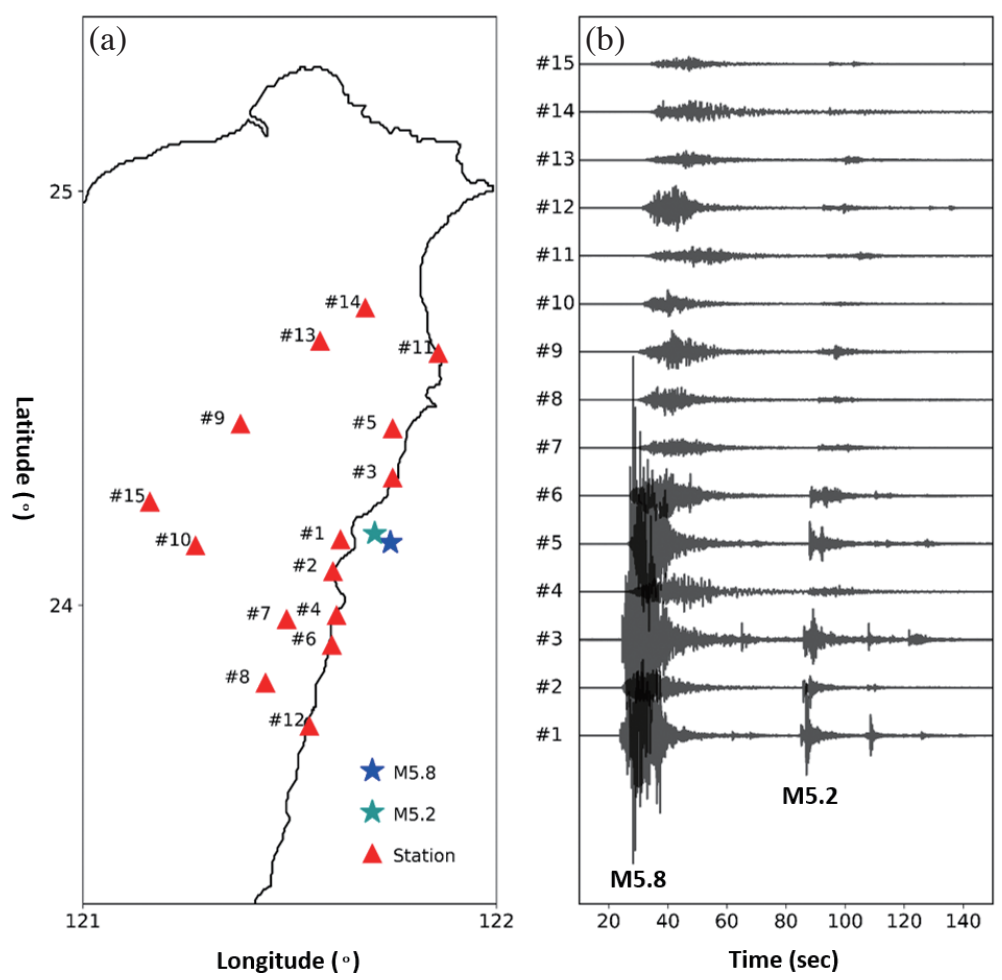

Fig. 8. Multi-event seismograms. (a) Red triangles show stations recorded seismograms of two events, and two stars show the epicenters of the two events, (b) seismograms recorded by stations in (a).

\subsection{Data and Resources}

Records used in this study were collected from the Central Weather Bureau Seismic Network (CWBSN) of Taiwan. Access to the waveforms records can be obtained from the Owners on request (http://www.cwb.gov.tw/eng/ index.htm).

Acknowledgements The software programs Earthworm (Johnson et al. 1995) and GMT (Wessel and Smith 1998) were used in this study and are gratefully acknowledged. Our work was supported by the Ministry of Science and Technology, Taiwan.

\section{REFERENCES}

Allen, R. V., 1978: Automatic earthquake recognition and timing from single traces. Bull. Seismol. Soc. Am., 68, 1521-1532.

Allen, R. M., P. Gasparini, O. Kamigaichi, and M. Böse, 2009: The status of earthquake early warning around the world: An introductory overview. Seismol. Res. Lett., 80, 682-693, doi: 10.1785/gssrl.80.5.682. [Link]

Chen, D.-Y., 2015: Development and study of Earthworm platform for earthquake early warning in Taiwan. Ph.D. Thesis, National Taiwan University, Taipei City, Taiwan, ROC, 119 pp.

Chen, D.-Y., T. L. Lin, Y.-M. Wu, and N.-C. Hsiao, 2012:
Testing a $P$-wave earthquake early warning system by simulating the 1999 Chi-Chi, Taiwan, $M_{\mathrm{w}} 7.6$ earthquake. Seismol. Res. Lett., 83, 103-108, doi: 10.1785/ gssrl.83.1.103. [Link]

Chen, D.-Y., N.-C. Hsiao, and Y.-M. Wu, 2015: The Earthworm based earthquake alarm reporting system in Taiwan. Bull. Seismol. Soc. Am., 105, 568-579, doi: 10.1785/0120140147. [Link]

Chen, D.-Y., Y.-M. Wu, and T.-L. Chin, 2017: An empirical evolutionary magnitude estimation for early warning of earthquakes. J. Asian Earth Sci., 135, 190-197, doi: 10.1016/j.jseaes.2016.12.028. [Link]

Geiger, L., 1912: Probability method for the determination of earthquake epicenters from the arrival time only. Bull. St. Louis Univ., 8, 60-71.

Hsiao, N.-C., 2006: The application of real-time strongmotion observations on the earthquake early warning in Taiwan. Ph.D. Thesis, National Central University, Taoyuan City, Taiwan, ROC, 178 pp.

Hsiao, N.-C., Y.-M. Wu, L. Zhao, D.-Y. Chen, W.-T. Huang, K.-H. Kuo, T.-C. Shin, and P.-L. Leu, 2011: A new prototype system for earthquake early warning in Taiwan. Soil Dyn. Earthq. Eng., 31, 201-208, doi: 10.1016/j.soildyn.2010.01.008. [Link]

Johnson, C. E., A. Bittenbinder, B. Bogaert, L. Dietz, and W. Kohler, 1995: Earthworm: A flexible approach to seismic network processing. IRIS Newsletter, 14, 1-4. 
Kodera, Y., J. Saitou, N. Hayashimoto, S. Adachi, M. Morimoto, Y. Nishimae, and M. Hoshiba, 2016: Earthquake early warning for the 2016 Kumamoto earthquake: Performance evaluation of the current system and the next-generation methods of the Japan Meteorological Agency. Earth Planets Space, 68, doi: 10.1186/s40623-016-0567-1. [Link]

Kuyuk, H. S., S. Colombelli, A. Zollo, R. M. Allen, and M. O. Erdik, 2015: Automatic earthquake confirmation for early warning system. Geophys. Res. Lett., 42, 5266-5273, doi: 10.1002/2015GL063881. [Link]

Lin, T. L. and Y. M. Wu, 2010: Magnitude estimation using the covered areas of strong ground motion in earthquake early warning. Geophys. Res. Lett., 37, L09301, doi: 10.1029/2010GL042797. [Link]

Lin, T. L., Y. M. Wu, and D. Y. Chen, 2011: Magnitude estimation using initial $\mathrm{P}$-wave amplitude and its spatial distribution in earthquake early warning in Taiwan. Geophys. Res. Lett., 38, L09303, doi: 10.1029/2011GL047461. [Link]

Rau, R.-J. and W.-T. Liang, 2017: Introduction to the special issue on the 2016 Meinong, Taiwan, earthquake. Terr. Atmos. Ocean. Sci., 28, I-III, doi: 10.3319/ TAO.2017.06.07.01. [Link]

Shin, T.-C. and T. Teng, 2001: An overview of the 1999 Chi-Chi, Taiwan, earthquake. Bull. Seismol. Soc. Am., 91, 895-913, doi: 10.1785/0120000738. [Link]
Teng, T., L. Wu, T.-C. Shin, Y.-B. Tsai, and W. H. K. Lee, 1997: One minute after: Strong-motion map, effective epicenter, and effective magnitude. Bull. Seismol. Soc. Am., 87, 1209-1219.

Wessel, P. and W. H. F. Smith, 1998: New, improved version of Generic Mapping Tools released. Eos, Trans., $A G U, 79,579$, doi: 10.1029/98EO00426. [Link]

Wu, Y.-M. and T. Teng, 2002: A virtual subnetwork approach to earthquake early warning. Bull. Seismol.Soc. Am., 92, 2008-2018, doi: 10.1785/0120010217. [Link]

Wu, Y.-M. and L. Zhao, 2006: Magnitude estimation using the first three seconds P-wave amplitude in earthquake early warning. Geophys. Res. Lett., 33, L16312, doi: 10.1029/2006g1026871. [Link]

Wu, Y.-M., J.-K. Chung, T.-C. Shin, N.-C. Hsiao, Y.-B. Tsai, W. H. K. Lee, and T. Teng, 1999: Development of an integrated earthquake early warning system in Taiwan - Case for the Hualien area earthquakes. Terr. Atmos. Ocean. Sci., 10, 719-736, doi: 10.3319/ TAO.1999.10.4.719(T). [Link]

Wu, Y.-M., J. B. H. Shyu, C.-H. Chang, L. Zhao, M. Nakamura, and S.-K. Hsu, 2009: Improved seismic tomography offshore northeastern Taiwan: Implications for subduction and collision processes between Taiwan and the southernmost Ryukyu. Geophys. J. Int., 178, 1042-1054, doi: 10.1111/j.1365-246x.2009.04180.x. [Link] 